\title{
A review on primary progressive aphasia
}

\author{
Gabriel C Léger ${ }^{1,2}$ \\ Nancy Johnson ${ }^{3}$ \\ 'Neurology Service, Hôtel-Dieu du \\ Centre Hospitalier de l'Univertité de \\ Montréal, Montréal, Québec, Canada; \\ ${ }^{2}$ Faculty of Medicine, Université de \\ Montréal, Montréal, Québec, Canada; \\ ${ }^{3}$ Cognitive Neurology and Alzheimer's \\ Disease Center, Department of \\ Psychiatry and Behavioral Science, \\ Northwestern University Feinberg \\ School of Medicine, Chicago, IL, USA
}

\begin{abstract}
Primary progressive aphasia (PPA) is a neurodegenerative disease of insidious onset presenting with progressive isolated loss of language function, without significant impairment in other cognitive domains. Current diagnostic criteria require the language dysfunction to remain isolated for at least two years, and to remain the salient feature as the disease progresses, usually to involve other domains such as behavior, executive functions, and judgment. Although PPA in its early stages can usually be differentiated from probable Alzheimer's disease (PRAD) and the behavioral variant of frontotemporal lobar degeneration by the absence of significant changes in memory and behavior, and the preservation of activities daily living, progression of the disease often leads to deficits more consistent with the latter. Underlying etiologies remain heterogeneous: the neuropathological characteristics associated with frontotemporal lobar degeneration, cortocobasal degeneration, and motor neuron disease are usually found. There is a strong genetic susceptibility with affliction of first-degree relatives with similar disease in up to 40 to $50 \%$ in some series. Pathogenic mutations in genes coding for the proteins tau and progranulin have been isolated. These are leading to a better understanding of the neuropathological mechanisms and hopefully targeted disease-modifying therapy. Current therapy is limited to improving mood symptoms and targeting behavior changes as they develop. Referral to specialized centers where speech therapy, counseling, and education for both patient and caregiver are available may be helpful.
\end{abstract}

Keywords: primary progressive aphasia

\section{Introduction}

Primary progressive aphasia (PPA) is a clinical syndrome defined by the insidious onset and progressive dissolution of language skills (Mesulam 1982, 2001, 2003). Although additional cognitive symptoms may emerge later in the course of illness, deficits must be grossly confined to the area of language for at least 2 years to fulfill the criteria for a diagnosis of PPA. The first description of the syndrome is attributed to Mesulam (1982), but reports of similar clinical findings have been in the literature for over 100 years (Mesulam and Weintraub 1992).

The neuropathological underpinnings of PPA are varied (see section on neuropathology), but the most frequent etiology may be one of the pathological processes leading to frontotemporal lobar degeneration (FTLD). As defined by Neary et al (1998), FTLD may present clinically as either a behavioral disorder, called frontotemporal dementia (FTD) or occasionally behavioral variant FTD (bv-FTD), or one of two so called language disorders: semantic dementia (SD) and progressive non-fluent aphasia (PNFA). These disorders and their relation to PPA will be discussed.

\section{Differential diagnosis}

PPA can be usually be differentiated from the clinical syndrome of Probable Alzheimer's Disease (PRAD) by the relative preservation of memory, and from FTD by the relative sparing of frontal lobe functions and appropriateness of behavior.

Although the language disorder in PPA may interfere with the ability to memorize word lists or solve reasoning tasks, the patient typically has no difficulty recalling daily 
events or behaving with good judgment, indicating that explicit memory, executive functions and social skills remain intact.

\section{Clinical presentation and evolution}

By definition, language disturbances are the most salient feature in the early clinical picture of PPA. In fact, the diagnostic criteria require at least two years of relatively isolated language impairment with generally intact functioning in other cognitive abilities such as episodic memory, visuospatial skills, reasoning, and comportment (Mesulam and Weintraub 1992). It may often be difficult to confirm normal function in other cognitive when significant language deficits are present. Modification of standard testing paradigms to reduce their dependence on language skills can be useful (Wicklund et al 2004). Deficits in other cognitive domains eventually emerge after the initial few years, but the language dysfunction remains the most salient feature and advances most rapidly, throughout the course of the illness (Mesulam 2003). Primary progressive aphasia is distinct from states of pure progressive dysarthria or phonological disintegration where the articulation rather than usage of words becomes disrupted. Patients with PPA may also show mild ideomotor (usually buccofacial) apraxia, dyscalculia, disinhibition, and constructional deficits (Joshi et al 2003). These additional symptoms indicate a progression or spread of dysfunction to prefrontal and parietal cortices immediately adjacent to the language network. The term PPA-plus has been proposed to designate this advancing state (Mesulam 2001).

PPA may be considered a syndromic entity that results from any one of a number of initially focal neuropathological processes, beginning in the language network (Kertesz et al 1994) (see section VI). With progression, these processes lead to more generalized clinical involvement resulting in recognizable diseases that can additionally include the behavioral variant of frontotemporal lobar degeneration, corticalbasal degeneration (CBD) (Kertesz et al 1994; Ikeda et al 1996; Gorno-Tempini, Murray et al 2004; McMonagle et al 2006), and even amyotrophic lateral sclerosis (ALS) (Bak et al 2001; Caselli et al 1993). Occasionally, Alzheimer's disease (Pogacar and Williams 1984; Greene et al 1990, 1996) or Creutzfeldt-Jakob disease (Shuttleworth et al 1985; Mandell et al 1989; Ghorayeb et al 1998) is diagnosed. Although the neurological examination may be completely normal in the early stages, the presence of increased muscle tone, apraxia, or subtle signs of parkinsonism may be harbingers of what is to follow. As PPA progresses, it blends into the symptomatology of FTD, CBD or the motor neuron disease of ALS so that significant dysarthria, primitive reflexes (particularly from the dominant hemisphere), hemiparesis, tremor, rigidity, bradykinesia or akinesia, alien hand phenomenon, hemisensory deficits, and fasciculations may be observed. Regardless of the underlying etiology, the unique feature of PPA is the initial predominance of the language deficit and the corresponding initial selectivity of the neurodegeneration for the language network.

\section{Subtypes of PPA}

Although he included detailed descriptions of the nature and evolution of the aphasia in each of his original 6 patients, Mesulam did not attempt to divide PPA into subgroups. Instead, he emphasized the remarkable selectivity that the disease seemed to demonstrate for the language network, regardless of the exact nature of the aphasia. Attempts to use traditional aphasia subtypes such as Broca's, Wernicke's, or Conduction Aphasia to classify language deficits in PPA have not been entirely successful (Clark et al 2005; George and Mathuranath 2005). Unlike aphasia due to stroke where the lesions tend to be more severe in a focal brain region, in PPA the degeneration is less severe and more widespread and the clinical symptoms are quite variable (Thompson et al 1997) and most do not conform to the traditional anatomical patterns based on stroke subjects. These difficulties notwithstanding, with the increasing number of cases being evaluated in detail, it has been possible for many groups to propose classification systems. These systems vary considerably in their complexity. The simplest of these follows Mesulam's contention that PPA is a single disease entity showing a smooth clinical spectrum spread over a continuum, with fluent, grammatically intact, but content poor speech reminiscent of Wernike's aphasia at one extreme, and non-fluent, agrammatic, effortful, and content rich speech, more in keeping with Broca's aphasia, at the other. Other investigators have proposed classifications based on at least three (Snowden et al 1992; Grossman and Ash 2004), and up to six (Kertesz et al 2003) categories. All present with and are characterized by the presence of anomia, and differentiated on the basis of measures of fluency, the presence or absence of logopenia or word finding difficulties, and loss of word meaning or comprehension. Part of the difficulty in classifying presentations of PPA lies in the nature of the disease process in any given individual, which begins with a distinct 'anatomy of disease onset' and evolves according to a variable 'progression trajectory'(Mesulam 2001). 
It is not surprising that there exists some controversy concerning the most natural approach to categorizing the progressive aphasias. An optimal classification system applicable during life would be clinically relevant, betray the underlying disease process, and predict its evolution. Classifying the presentation helps localize the brain areas initially affected and, as the extent of the pathology progresses to involve neighboring systems, predicts the manifestations to follow.

In addition to the clinical criteria for the diagnosis of the behavioral presentation of FTD (bvFTD), Neary et al (1998), in their description of the possible clinical entities resulting from the pathological processes involved in FTLD, also distinguished two forms of language presentations: PNFA and SD. The term SD (Hodges et al 1992; Snowden et al 1992; Neary et al 1998) was introduced to designate a unique syndrome in which a prominent fluent aphasia with impaired comprehension emerges in the presence of prominent deficits of visual recognition (agnosia). There is also belief that patients with SD can be shown to have circumscribed deficits in semantic knowledge that contributes to their language difficulties (Snowden et al 1989; Hodges et al 1992). In clinical practice, this term has also been used to denote patients with a fluent aphasia and verbal comprehension deficits, even in the absence of visual processing deficits. The term PNFA denotes patients with a non-fluent aphasia and relatively preserved comprehension. Patients with PNAF and non-agnostic SD would also fulfill criteria for a diagnosis of PPA. Because fluency and grammaticism are the most clinically obvious differences between these two presentations, the term 'fluent PPA' is sometimes equated to SD, and 'non-fluent PPA' or 'agrammatic PPA' to PNFA.

There has evolved an extensive literature comparing various subtypes of PPA in terms of the associated cognitive and behavioral manifestations, structural and functional imaging findings, and pathological underpinnings. Although a complete analysis of this literature is beyond the scope of this short review, key references will be made within relevant sections.

Gorno-Tempini, Dronkers et al (2004) described three subtypes of PPA, logopenic, agrammatic, and semantic, which correspond to distinctive patterns of brain atrophy. The logopenic variant is characterized by word-finding difficulties and decreased output, but relatively preserved syntax, grammar and comprehension. The agrammatic subtype, which is similar to the Neary et al (1998) criteria for the PNFA subtype of FTLD, is characterized by labored speech, agrammatism in production and/or comprehension, variable degrees of anomia, and phonemic paraphasias, in the presence of relatively normal word comprehension. The semantic variant is characterized by fluent, grammatically correct speech, loss of word and object meaning, surface dyslexia and relatively preserved syntactic comprehension skills.

Loss of semantic memory and non-verbal deficits in progressive aphasias were recognized initially by Warrington (1975). Deficits of this kind are found in patients with SD (Snowden et al 1989; Hodges et al 1992). Debate about the ability to confirm such deficits in patients with language impairment has generated experimental paradigms that rely more on pictures, such as the 'Pyramids and Palm Trees' test (Howard and Patterson 1992), which assesses semantic associations non-verbally, tasks involving coloring of black and white line drawing of well known animals, and on tests matching environmental sounds to target pictures (Bozeat et al 2000).

In a comprehensive review of the literature, Grossman and Ash (2004) conclude that there is enough evidence to support the notion that PPA can in fact be divided into PNFA and SD (without visual agnosia). Their conclusions are based on an analysis of both the linguistic profiles and imaging characteristics of these variants. In a novel data driven hierarchical cluster analysis of the clinical characteristics of patients with PPA, Knibb et al (2006) also confirmed the importance of two major presentations that roughly conform to these variants.

That PPA can present in fluent or non-fluent forms is clear; however, Mesulam has argued that the use of the term $\mathrm{SD}$, to denote the fluent form of PPA is not entirely accurate because it should, as defined by Neary (1998), include a visual associative agnosia, and is therefore not strictly a language disorder (Weintraub et al 1990; Mesulam 2001, 2003). He has advocated the use of the term SD only in the context of demonstrable agnosia and suggested that SD must represent an affliction of at least two separate systems (Mesulam 2001). In response, Adlam et al (2006) have described seven patients who met Mesulams criteria for the diagnosis of PPA (Mesulam 2001, 2003), but additionally had subtle non-verbal deficits in semantic memory when these were expressly sought using sensitive paradigms that they developed. Although there still remains much debate concerning the use of the term SD to describe what is initially perceived as a 'pure' language disorder, its historic impact and commonplace usage has made it important to discuss.

The US National Alzheimer's Coordinating Center (NACC), in an effort to standardize data collection and 
diagnostic criteria for Alzheimer's disease and related dementias, has collated complete lists of diagnostic criteria, with relevant references. In their treatment of the progressive aphasias, they recommend the use of the criteria defined by Mesulam, but further subcategorize according to the Neary, into PNFA and SD, with special attention to the presence or absence of agnosia associated with SD, and add a fourth category 'other' that includes logopenic, anomic, transcortical, word deafness, syntactic comprehension, and motor speech disorder (https://www.alz.washington.edu/NONMEMBER/ UDS/DOCS/VER1_2/pubguide.pdf, p 54-8).

\section{Behavior, emotion, and mood}

Although not a salient feature of PPA in its early stages (Weintraub et al 1990), the emergence of behavioral changes generally signal progression of the disease, as has been quantified using various measures including Kertesz' Frontal Behavioral Inventory (FBI) (Kertesz et al 1997; Marczinski et al 2004) and the NPI-Q (Kaufer et al 2000; Banks and Weintraub, in press). The types of behavioral changes that emerge seem to depend on the aphasia variant at hand and reflect progression of the disease from the variants epicenter to adjacent areas. In this way, patients with SD were found to develop apathy and changes in food preferences, while patients with a non-fluent form exhibited later onset irritability (Snowden et al 1992). Stereotypic behavior, changes in eating preference, disinhibition and reduced social awareness can also be demonstrated as earlier features of SD (Bozeat et al 2000).

Depression in PPA is being recognized (Medina and Weintraub, in press), perhaps as a reaction to their ongoing losses and preserved insight (Eslinger et al 2005). Depressive symptoms such as irritability seem to remain present for longer periods (Kertesz et al 2000).

\section{Imaging}

Patients with PPA typically show gyral atrophy of the left hemisphere involving frontal, temporal, insular and parietal components of the language network (Mesulam and Weintraub 1992; Rosen, Kramer et al 2002). In PPA, metabolic abnormalities tend to parallel predominant language dysfunction, in that non-fluent patients have reduced metabolism in left frontal areas, including the insula, while fluent patients with impairments in comprehension tend to have reduced left temporal metabolism (Abe et al 1997; Rosen, Gorno-Tempini et al 2002; Nestor et al 2003).

With regard to specific subtypes, Gorno-Tempini, Dronkers et al (2004) used voxel-based morphometry (VBM) to demonstrate the differential involvement of distinct brain regions in the three variants that they defined clinically. In PNFA, atrophy involved the left inferior frontal and insular cortex. In their semantic variant, the anterior temporal regions were preferentially affected, while in the logopenic patients, involved areas were the left posterior temporal and inferior parietal cortices. The same group also demonstrated a relationship between the appearance of mutism in PNFA and further atrophy of the pars opercularis and extension into the left basal ganglia (Gorno-Tempini et al 2006). Bilateral temporal atrophy, left greater than right, has also previously been demonstrated in SD by Mummery et al (2000) and Rosen, Gorno-Tempini et al (2002). In their report, Mummery et al (2000) also demonstrated a relationship between the degree of semantic impairment and the extent of left anterior temporal lobe atrophy. Rosen, Kramer et al (2002) had also previously demonstrated similar changes in their report of 3 patients with variants of PPA. In their combined PET and VBM analysis of patients with PNFA, Nestor et al (2003) demonstrated hypometabolism in the left anterior insula/frontal opercular region, and atrophy in the left peri-Sylvian region. PET and SPECT imaging may be able to differentiate the underlying etiology of PNFA (Nestor et al 2007). When only Alzheimer's disease-like changes (see pathology section below) were found at autopsy, nuclear imaging during the initial diagnostic workup more consistently demonstrated hypoperfusion deficits in the posterior temporoparietal association cortex, a pattern very specific for PRAD, in contrast to changes in the left anterior insula/ frontal opercular region noted above that are associated with 'FTD-spectrum' pathology (Nestor et al 2007).

Although often present with aphasia, the development of apraxia of speech suggests involvement beyond the language network. Using VBM, Josephs et al (2006) demonstrated that the presence of non-fluent aphasia was associated with anterior peri-sylvian atrophy, while the presence of speech apraxia followed changes in left premotor and supplemental motor cortex.

Despite marked left hemisphere dysfunction in non-fluent PPA, the metabolic state of the contralateral right hemisphere tends to remain within the normal range (Chawluk et al 1986; Tyrrell et al 1990). When asked to identify homonyms or synonyms in the course of functional MRI (fMRI) experiments, PPA patients (fluent and nonfluent) and controls activate the same components of the language network (Sonty et al 2003). However, in contrast to neurologically intact subjects, the PPA patients display aberrant (possibly compensatory) activations within 
regions of the brain outside of the classic language network (Mummery et al 1999; Sonty et al 2003). Using fMRI and a grammatically complex sentence comprehension paradigm, Cooke et al (2003) showed that patients with PNFA produce less activation within the ventral portion of the inferior frontal cortex, an area felt to be crucial to the processing of complex sentences. They further postulate that this interruption of a putative large-scale neural network for sentence comprehension (Cooke et al 2006) forms the basis for the complex grammatic comprehension deficits seen in these patients.

\section{Neuropathology}

Generally, as a group, the various neuropathological findings associated with PPA and FTD have been called frontotemporal lobar degeneration (FTLD) (Neary et al 1998). These are not uniform and their characterization is changing as a result of improvements in genetics and staining techniques. Specifically, the neuropathological findings are a function of a number of possible underlying etiologies. At autopsy, the most uniform finding is that of focal degeneration, which is usually found preferentially and more intensely in language areas, and characterized by neuronal loss, gliosis, and spongiform changes involving the superficial cortical layers. Cortex may also contain occasional ballooned neurons, called Pick cells. Staining with silver and newer histochemical stains for either the microtubule associated protein tau (MAPT or simply tau) or ubiquitin can reveal neuronal and glial inclusions in a pattern particular to the exact neurpathological process at hand. Pick bodies are argyrophilic (silver staining) spherical neuronal inclusions diagnostic of Pick's disease. Similar neuronal inclusions may only be visible with tau immunostaining, thus defining the underlying disorder as a 'tauopathy' such as in certain forms of FTD, CBG, and progressive supranuclear palsy (PSP). Other non-argyrophilic tau negative inclusions may stain only for ubiquitin, as is found when FTD is associated with motor neuron disease. When ubiquitin inclusions are found in the absence of clinical motor neuron disease, the finding is termed FTD-MND type. FTD associated with ubiquitin inclusions is also referred to as FTLD-U. When no inclusions can be found, the pathology is often referred to as dementia lacking distinctive histopathology (DLDH) (Knopman et al 1990), a diagnosis that is becoming increasingly scarce as immunohistochemical techniques are refined (Mackenzie et al 2006). Although these pathological findings are quite distinct in their staining characteristics and even genetic underpinnings (see below), they are generally considered as representing the 'FTD-spectrum' of pathologies that are most frequently found in FTLD.

Occasionally extracellular plaques and neuronal neurfibrillary tangles characteristic of Alzheimer's disease are found. In many instances their distribution is unusual and follows a more frontotemporal pattern, rather than the classic temporparietal one, resulting in brains with a macroscopic appearance more in keeping with FTLD (Knibb et al 2006).

A recent clinicopathological study (Forman et al 2006) suggests that tau pathology is more frequently associated with extrapyramidal disorders while FTLD-U manifests more frequently as bv-FTD, PPA, and motorneuron disease. In their cluster analysis, Knibb et al (2006) found AD-like changes accounted for roughly one third cases of fluent and non-fluent PPA. They also noted that tau-based disease was more common than FTLD-U in the non-fluent cluster (43 vs 17\%), while in the fluent group this trend was reversed (13 vs $53 \%)$.

\section{Genetics}

The not uncommon familial association of PPA and FTD supports a genetic link between them. Some 38 to $50 \%$ of clinical FTD cases appear to be familial (Stevens et al 1998; Grasbeck et al 2005). Countless kindred have been described that demonstrate segregation of a disease in an autosomal dominant pattern of inheritance. The clinical disease itself is usually FTD, but can include parkinsonism, motor neuron disease, or progressive aphasia, even in the same family. In some families, a mutation in the gene coding for tau (found on chromosome 17q21) can be demonstrated. Postmortem analysis usually reveals tau pathology.

More recently, a genetic cause of FTD with tau-negative ubiquinated inclusions (FTLD-U) was traced to progranulin $(P R G N)$ gene mutations, also found on chromosome 17q21 (Cruts et al 2006; Baker et al 2006). The mutations cause a null allele and a reduction in the level of functional $P R G N$ protein. Replacement of this missing protein may represent a new and exciting therapeutic avenue for this group of disorders. Snowden and colleagues have reported an increased occurrence of PNFA variant of progressive aphasia associated with mutations of this gene (Snowden et al 2006). Josephs et al (2007) have also noted more frequent language impairment in patients with FTLD-U associated with this mutation than patients without. These patients carried an initial diagnosis of PPA and developed early mutism.

Although the actual gene mutations have not yet been described, other families with related clinical disorders demonstrate linkage to different regions of chromosome 3 
(Brown et al 1995, 2004; Gydesen et al 2002) and 9 (Hosler et al 2000; Kovach et al 2001; Morita et al 2006).

In most families described above, the PPA is either quickly followed by the appearance of deficits in other cognitive domains, or other affected family members do not have "typical" PPA, and do not strictly meet the criteria of Weintraub et al (1990) and Mesulam (2001). However, Krefft and colleagues (2003) describe a family in which all three affected siblings meet Mesulam's more stringent criteria. The genetic basis for this kindred, and another in which the affected siblings also meet these criteria, have recently been shown to be a result of two new PRGN mutations (Mesulam et al 2007).

\section{Risk factors}

Certain genetic risk factors have also been noted. These include heterozygocity for codon 129 of the prion protein (Li et al 2005), presence of the tau H1/H1 genotype, also found to be associated with PSP and CBD (Li et al 2005), and ApoE $\varepsilon 2-\varepsilon 4$ hereozygocity (Acciarri et al 2006). Vasectomy may also increase the risk of developing PPA (Weintraub et al 2006). The exact mechanism for this remains unknown, but may involve a vasectomy induced immune response to sperm that share antigenic epitopes with brain.

\section{Treatment}

Treatment of PPA should be considered along at least three different avenues: (1) pharmacologic treatments to address associated symptoms and potentially slow or stop progression, (2) non-pharmacologic interventions to help maintain independent function and compensate for growing language deficits, and (3) support and education of patients and their care givers.

Because of the uncommon nature of PPA, there have been no large-scale clinical trials using any known pharmacologic agents. The only published therapy trial in PPA is that of Reed and colleagues (2004), who could not demonstrate a benefit of the dopamine agonist bromocriptine in their small, randomized, placebo-controlled trial.

A few clinical trials, as well as prospective and retrospective case series have been published using various pharmacological agents in patients with FTD (Chow 2005; Huey et al 2006; Freedman 2007). Conclusions from these studies become more relevant to PPA as the disorder progresses and behavioral manifestations become more prominent. The use of antidepressants such as trazadone seem to help stabilize the behavioral symptoms, but, unfortunately, without an impact on cognition (Lebert et al 2004). Similar benefits have been found with the cholinesterase inhibitor rivastigmine (Moretti et al 2004), and the MAO-B inhibitor selegiline (Moretti et al 2002).
In a novel application of a research tool that is finding more frequent use in neurological and psychiatric disorders, Finocchiaro and colleagues (2006) have demonstrated that high-frequency repetitive transcranial magnetic stimulation (hf-rTMS) applied to the left prefrontal cortex produces a significant and lasting improvement on language tasks, including verb production. The exact mechanism of such an improvement remains unknown, but it is believed that hf-rTMS may strengthen neural connection in those areas weakened by the disease process.

As has been demonstrated by Medina and Weintraub (in press), the frustration of progressive language deficits, particularly when insight is preserved, can lead to depressed mood. Evaluation and treatment of depression with routine antidepressants is indicated.

Disease modifying agents are not yet commercially available, but potential application of tau reducing drugs presently being developed for Alzheimer's disease may prove beneficial in PPA patients with tau-based disease (Klafki et al 2006). When these become available, the need to distinguish tau from non-tau based disease during life will become important, and may be aided by genetic analysis, new PET imaging ligands (Small et al 2004, 2006), and cerebrospinal fluid tau species and levels (Hampel and Teipel 2004). Once the neuropathological mechanism involved in null progranulin-mutation based disease is better understood, these patients will likely benefit from some form of replacement therapy.

The frustration and helplessness experienced by family and caregivers should not be overlooked. Counseling and education are key. Although families can find much information on the FTD website (http://www.ftd-picks.org) and books (Radin et al 2004), some centers have developed education and support seminars series that have been received very positively (Diehl et al 2003; Banks et al 2006).

Finally, notwithstanding the progressive nature of PPA, there remains a role for regular speech therapy assessments, which can identify the impact of particular language deficits on day-to-day function, and help with the implementation of evolving communication strategies to help compensate (Snowden et al 1995; Graham et al 1999; Galton et al 2001; Jokel et al 2002, 2006). The detection of swallowing difficulties is also important as appropriate changes in diet and feeding habits could be lifesaving.

\section{Conclusion}

Since its original description by Mesulam in 1982, our understanding of the clinical evolution and neuropatholgic and genetic 
basis of PPA has progressed steadily. PPA should be considered in the differential diagnosis of dementias presenting with language dysfunction. It can be differentiated from PRAD and FTD by the initial absence of significant memory loss or behavioral changes, and the preservation of activities of daily living.

There exists presently no effective treatment for this condition, although addressing mood and behavioral issues, as well as helping caregivers better understand the disease is useful. As the mechanisms leading to the related diseases FTD, CBD, and ALS are unraveled, and pathology targeted therapy is developed, the future is more promising.

\section{References}

Abe K, Ukita H, Yanagihara T. 1997. Imaging in primary progressive aphasia. Neuroradiology, 39:556-9.

Acciarri A, Masullo C, Bizzarro A, et al. 2006. ApoE epsilon2-epsilon4 genotype is a possible risk factor for primary progressive aphasia. Ann Neurol, 59:436-7.

Adlam AL, Patterson K, Rogers TT, et al. 2006. Semantic dementia and fluent primary progressive aphasia: two sides of the same coin? Brain, 129:3066-80.

Bak TH, O'donovan DG, Xuereb JH, et al. 2001. Selective impairment of verb processing associated with pathological changes in Brodmann areas 44 and 45 in the motor neurone disease-dementia-aphasia syndrome. Brain, 124:103-20.

Baker M, Mackenzie IR, Pickering-Brown SM, et al. 2006. Mutations in progranulin cause tau-negative frontotemporal dementia linked to chromosome 17. Nature, 442:916-19.

Banks S, Rogalski E, Medina J, et al. 2006. Organizing a series of education and support conferences for caregivers of individuals with frontotemporal dementia and primary progressive aphasia. Alzheimer's Care Quarterly, 7:243-50.

Banks S, Weintraub S. In press. Neuropsychiatric symptoms in behavioral variant frontotemporal dementia and primary progressive aphasia. Journal of Geriatric Psychiatry and Neurology.

Bozeat S, Lambon Ralph MA, Patterson K, et al. 2000. Non-verbal semantic impairment in semantic dementia. Neuropsychologia, 38:1207-15.

Brown J, Ashworth A, Gydesen S, et al. 1995. Familial non-specific dementia maps to chromosome 3. Hum Mol Genet, 4:1625-8.

Brown J, Gydesen S, Johannsen P, et al. 2004. Frontotemporal dementia linked to chromosome 3. Dement Geriatr Cogn Disord, 17:274-6.

Caselli RJ, Windebank AJ, Petersen RC, et al. 1993. Rapidly progressive aphasic dementia and motor neuron disease. Ann Neurol, 33:200-7.

Chawluk JB, Mesulam MM, Hurtig H, et al. 1986. Slowly progressive aphasia without generalized dementia: studies with positron emission tomography. Ann Neurol, 19:68-74.

Chow TW. 2005. Treatment approaches to symptoms associated with frontotemporal degeneration. Curr Psychiatry Rep, 7:376-80.

Clark DG, Charuvastra A, Miller BL, et al. 2005. Fluent versus nonfluent primary progressive aphasia: a comparison of clinical and functional neuroimaging features. Brain Lang, 94:54-60.

Cooke A, Devita C, Gee J, et al. 2003. Neural basis for sentence comprehension deficits in frontotemporal dementia. Brain Lang, 85:211-21.

Cooke A, Grossman M, Devita C, et al. 2006. Large-scale neural network for sentence processing. Brain Lang, 96:14-36.

Cruts M, Gijselinck I, Van Der Zee J, et al. 2006. Null mutations in progranulin cause ubiquitin-positive frontotemporal dementia linked to chromosome 17q21. Nature, 442:920-4.

Diehl J, Mayer T, Kurz A, et al. 2003. [Features of frontotemporal dementia from the perspective of a special family support group]. Nervenarzt, 74:445-9.

Eslinger PJ, Dennis K, Moore P, et al. 2005. Metacognitive deficits in frontotemporal dementia. J Neurol Neurosurg Psychiatry, 76:1630-5.
Finocchiaro C, Maimone M, Brighina F, et al. 2006. A case study of primary progressive aphasia: improvement on verbs after rTMS treatment. Neurocase, 12:317-21.

Forman MS, Farmer J, Johnson JK, et al. 2006. Frontotemporal dementia: clinicopathological correlations. Ann Neurol, 59:952-62.

Freedman M. 2007. Frontotemporal dementia: recommendations for therapeutic studies, designs, and approaches. Can J Neurol Sci, 34 (Suppl 1):S118-24.

Galton CJ, Gomez-Anson B, Antoun N, et al. 2001. Temporal lobe rating scale: application to Alzheimer's disease and frontotemporal dementia. J Neurol Neurosurg Psychiatry, 70:165-73.

George A, Mathuranath PS. 2005. Primary progressive aphasia: a comparative study of progressive nonfluent aphasia and semantic dementia. Neurol India, 53:162-5; discussion 165-6.

Ghorayeb I, Series C, Parchi P, et al. 1998. Creutzfeldt-Jakob disease with long duration and panencephalopathic lesions: molecular analysis of one case. Neurology, 51:271-4.

Gorno-Tempini ML, Dronkers NF, Rankin KP, et al. 2004. Cognition and anatomy in three variants of primary progressive aphasia. Ann Neurol, 55:335-46.

Gorno-Tempini ML, Murray RC, Rankin KP, et al. 2004. Clinical, cognitive and anatomical evolution from nonfluent progressive aphasia to corticobasal syndrome: a case report. Neurocase, 10:426-36.

Gorno-Tempini ML, Ogar JM, Brambati SM, et al. 2006. Anatomical correlates of early mutism in progressive nonfluent aphasia. Neurology, 67:1849-51.

Graham KS, Patterson K, Pratt KH, et al. 1999. Relearning and subsequent forgetting of semantic category exemplars in a case of semantic dementia. Neuropsychology, 13:359-80.

Grasbeck A, Horstmann V, Nilsson K, et al. 2005. Dementia in first-degree relatives of patients with frontotemporal dementia. A family history study. Dement Geriatr Cogn Disord, 19:145-53.

Greene J, Morris JC, Sandson J, et al. 1990. Progressive aphasia: a precursor of global dementia? Neurology, 40:423-9.

Greene JD, Patterson K, Xuereb J, et al. 1996. Alzheimer disease and nonfluent progressive aphasia. Arch Neurol, 53:1072-8.

Grossman M, Ash S. 2004. Primary progressive aphasia: a review. Neurocase, 10:3-18.

Gydesen S, Brown JM, Brun A, et al. 2002. Chromosome 3 linked frontotemporal dementia (FTD-3). Neurology, 59:1585-94.

Hampel H, Teipel SJ. 2004. Total and phosphorylated tau proteins: evaluation as core biomarker candidates in frontotemporal dementia. Dement Geriatr Cogn Disord, 17:350-4.

Hodges JR, Patterson K, Oxbury S, et al. 1992. Semantic dementia. Progressive fluent aphasia with temporal lobe atrophy. Brain, 115:1783-806.

Hosler BA, Siddique T, Sapp PC, et al. 2000. Linkage of familial amyotrophic lateral sclerosis with frontotemporal dementia to chromosome 9q21-q22. JAMA, 284:1664-9.

Howard D, Patterson K. 1992. Pyramids and palm trees: a test of semantic access from pictures and words. Bury St. Edmonds, Suffolk, UK: Thames Valley Test Company.

Huey ED, Putnam KT, Grafman J. 2006. A systematic review of neurotransmitter deficits and treatments in frontotemporal dementia. Neurology, 66:17-22.

Ikeda K, Akiyama H, Iritani S, et al. 1996. Corticobasal degeneration with primary progressive aphasia and accentuated cortical lesion in superior temporal gyrus: case report and review. Acta Neuropathol (Berl), 92:534-9.

Jokel R, Rochon E, Leonard C. 2002. Therapy for anomia in semantic dementia. Brain Cogn, 49:241-4.

Jokel R, Rochon E, Leonard C. 2006. Treating anomia in semantic dementia: improvement, maintenance, or both? Neuropsychol Rehabil, 16:241-56.

Josephs KA, Ahmed Z, Katsuse O, et al. 2007. Neuropathologic features of frontotemporal lobar degeneration with ubiquitin-positive inclusions with progranulin gene (PGRN) mutations. J Neuropathol Exp Neurol, $66: 142-51$. 
Josephs KA, Duffy JR, Strand EA, et al. 2006. Clinicopathological and imaging correlates of progressive aphasia and apraxia of speech. Brain, 129:1385-98.

Joshi A, Roy EA, Black SE, et al. 2003. Patterns of limb apraxia in primary progressive aphasia. Brain Cogn, 53:403-7.

Kaufer DI, Cummings JL, Ketchel P, et al. 2000. Validation of the NPI-Q, a brief clinical form of the Neuropsychiatric Inventory. J Neuropsychiatry Clin Neurosci, 12:233-9.

Kertesz A, Davidson W, Fox H. 1997. Frontal behavioral inventory: diagnostic criteria for frontal lobe dementia. Can J Neurol Sci, 24:29-36.

Kertesz A, Davidson W, Mccabe P, et al. 2003. Primary progressive aphasia: diagnosis, varieties, evolution. J Int Neuropsychol Soc, 9:710-19.

Kertesz A, Hudson L, Mackenzie IR, et al. 1994. The pathology and nosology of primary progressive aphasia. Neurology, 44:2065-72.

Kertesz A, Nadkarni N, Davidson W, et al. 2000. The frontal behavioral inventory in the differential diagnosis of frontotemporal dementia. $J$ Int Neuropsychol Soc, 6:460-8.

Klafki HW, Staufenbiel M, Kornhuber J, et al. 2006. Therapeutic approaches to Alzheimer's disease. Brain, 129:2840-55.

Knibb JA, Xuereb JH, Patterson K, et al. 2006. Clinical and pathological characterization of progressive aphasia. Ann Neurol, 59:156-65.

Knopman DS, Mastri AR, Frey WH, et al. 1990. Dementia lacking distinctive histologic features: a common non-Alzheimer degenerative dementia. Neurology, 40:251-6.

Kovach MJ, Waggoner B, Leal SM, et al. 2001. Clinical delineation and localization to chromosome 9p13.3-p12 of a unique dominant disorder in four families: hereditary inclusion body myopathy, Paget disease of bone, and frontotemporal dementia. Mol Genet Metab, 74:458-75.

Krefft TA, Graff-Radford NR, Dickson DW, et al. 2003. Familial primary progressive aphasia. Alzheimer Dis Assoc Disord, 17:106-12.

Lebert F, Stekke W, Hasenbroekx C, et al. 2004. Frontotemporal dementia: a randomised, controlled trial with trazodone. Dement Geriatr Cogn Disord, 17:355-9.

Li X, Rowland LP, Mitsumoto H, et al. 2005. Prion protein codon 129 genotype prevalence is altered in primary progressive aphasia. Ann Neurol, 58:858-64.

Mackenzie I, Shi J, Shaw C, et al. 2006. Dementia lacking distinctive histology (DLDH) revisited. Acta Neuropathol (Berl), 112:551-9.

Mandell AM, Alexander MP, Carpenter S. 1989. Creutzfeldt-Jakob disease presenting as isolated aphasia. Neurology, 39:55-8

Marczinski CA, Davidson W, Kertesz A. 2004. A longitudinal study of behavior in frontotemporal dementia and primary progressive aphasia. Cogn Behav Neurol, 17:185-90.

McMonagle P, Blair M, Kertesz A. 2006. Corticobasal degeneration and progressive aphasia. Neurology, 67:1444-51.

Medina J, Weintraub S. . . In press. Depression in primary progressive aphasia. Journal of Geriatric Psychiatry and Neurology.

Mesulam M, Johnson N, Krefft TA, et al. 2007. Progranulin mutations in primary progressive aphasia: the PPA1 and PPA3 families. Arch Neurol, 64:43-7.

Mesulam MM. 1982. Slowly progressive aphasia without generalized dementia. Ann Neurol, 11:592-8.

Mesulam MM. 2001. Primary progressive aphasia. Ann Neurol, 49:425-32.

Mesulam MM. 2003. Primary progressive aphasia - a language-based dementia. N Engl J Med, 349:1535-42.

Mesulam MM, Weintraub S. 1992. Spectrum of primary progressive aphasia. Baillieres Clin Neurol, 1:583-609.

Moretti R, Torre P, Antonello RM, et al. 2004. Rivastigmine in frontotemporal dementia: an open-label study. Drugs Aging, 21:931-7.

Moretti R, Torre P, Antonello RM, et al. 2002. Effects of selegiline on fronto-temporal dementia: a neuropsychological evaluation. Int J Geriatr Psychiatry, 17:391-2.

Morita M, Al-Chalabi A, Andersen PM, et al. 2006. A locus on chromosome 9p confers susceptibility to ALS and frontotemporal dementia. Neurology, 66:839-44.
Mummery CJ, Patterson K, Price CJ, et al. 2000. A voxel-based morphometry study of semantic dementia: relationship between temporal lobe atrophy and semantic memory. Ann Neurol, 47:36-45.

Mummery CJ, Patterson K, Wise RJ, et al. 1999. Disrupted temporal lobe connections in semantic dementia. Brain, 122:61-73.

Neary D, Snowden JS, Gustafson L, et al. 1998. Frontotemporal lobar degeneration: a consensus on clinical diagnostic criteria [see comments]. Neurology, 51:1546-54.

Nestor PJ, Balan K, Cheow HK, et al. 2007. Nuclear imaging can predict pathologic diagnosis in progressive nonfluent aphasia. Neurology, 68:238-9.

Nestor PJ, Graham NL, Fryer TD, et al. 2003. Progressive non-fluent aphasia is associated with hypometabolism centred on the left anterior insula. Brain, 126:2406-18.

Pogacar S, Williams RS. 1984. Alzheimer's disease presenting as slowly progressive aphasia. $R$ I Med $J, 67: 181-5$.

Radin L, Radin M, Grossman M. 2004. What if it's not Alzheimer's? A caregiver's guide to frontotemporal dementia Prometheus Books.

Reed DA, Johnson NA, Thompson C, et al. 2004. A clinical trial of bromocriptine for treatment of primary progressive aphasia. Ann Neurol, $56: 750$.

Rosen HJ, Gorno-Tempini ML, Goldman WP, et al. 2002. Patterns of brain atrophy in frontotemporal dementia and semantic dementia. Neurology, 58:198-208.

Rosen HJ, Kramer JH, Gorno-Tempini ML, et al. 2002. Patterns of cerebral atrophy in primary progressive aphasia. Am J Geriatr Psychiatry, 10:89-97.

Shuttleworth EC, Yates AJ, Paltan-Ortiz JD. 1985. Creutzfeldt-Jakob disease presenting as progressive aphasia. J Natl Med Assoc, 77:649-50, $652,655-6$.

Small GW, Kepe V, Ercoli LM, et al. 2006. PET of brain amyloid and tau in mild cognitive impairment. $N$ Engl J Med, 355:2652-63.

Small GW, Kepe V, Huang MC, et al. 2004. In vivo brain imaging of tau aggregation in frontal temporal dementia using [F-18]FDDNP positron emission tomography. 9th International Conference on Alzheimer's Disease and Related Disorders.

Snowden JS, Goulding PJ, Neary D. 1989. Semantic dementia: a form of circumscribed cerebral atrophy. Behavioral Neurology, 2:167-82.

Snowden JS, Griffiths HL, Neary D. 1995. Autobiographical experience and word meaning. Memory, 3:225-46.

Snowden JS, Neary D, Mann DM, et al. 1992. Progressive language disorder due to lobar atrophy. Ann Neurol, 31:174-83.

Snowden JS, Pickering-Brown SM, Mackenzie IR, et al. 2006. Progranulin gene mutations associated with frontotemporal dementia and progressive non-fluent aphasia. Brain, 129:3091-102.

Sonty SP, Mesulam MM, Thompson CK, et al. 2003. Primary progressive aphasia: PPA and the language network. Ann Neurol, 53:35-49.

Stevens M, Van Duijn CM, Kamphorst W, et al. 1998. Familial aggregation in frontotemporal dementia. Neurology, 50:1541-5.

Thompson C, Ballard K, Tait M, et al. 1997. Patterns of language decline in non-fluent primary progressive aphasia. Aphasiology, 11:297-321.

Tyrrell PJ, Warrington EK, Frackowiak RS, et al. 1990. Heterogeneity in progressive aphasia due to focal cortical atrophy. A clinical and PET study. Brain, 113:1321-36.

Warrington EK. 1975. The selective impairment of semantic memory. QJ Exp Psychol, 27:635-57.

Weintraub S, Fahey C, Johnson N, et al. 2006. Vasectomy in men with primary progressive aphasia. Cogn Behav Neurol, 19:190-3.

Weintraub S, Rubin NP, Mesulam MM. 1990. Primary progressive aphasia. Longitudinal course, neuropsychological profile, and language features. Arch Neurol, 47:1329-35.

Wicklund AH, Johnson N, Weintraub S. 2004. Preservation of reasoning in primary progressive aphasia: further differentiation from Alzheimer's disease and the behavioral presentation of frontotemporal dementia. $J$ Clin Exp Neuropsychol, 26:347-55. 